\title{
FACTORES ASOCIADOS AL AUSENTISMO VOLUNTARIO Y SU RELACIÓN CON EL RENDIMIENTO ACADÉMICO EN ESTUDIANTES DE MEDICINA DE LA UNIVERSIDAD DE PANAMÁ
}

\author{
FACTORS RELATED TO VOLUNTARY ABSENTEEISM AND ITS RELATIONSHIP WITH \\ ACADEMIC PERFORMANCE IN MEDICAL STUDENTS IN UNIVERSITY OF PANAMA
}

\author{
Bravo, Harold; Barrios, Maria; Barraza, Emar; Arrocha, David \\ * Estudiante del XII Semestre de Medicina, de la Universidad de Panamá \\ Recibido: 5 de noviembre del 2018 \\ Aceptado: 6 de octubre del 2019 \\ Bravo H, Barrios MA, Barraza E, Arrocha E. Factores Asociados al Ausentismo Voluntario y su Relación con el Rendimiento Académico en \\ Estudiantes de Medicina de la Universidad de Panamá. Rev méd cient. 2018;31:43-52.
}

\section{RESUMEN}

Introducción: El ausentismo estudiantil es la inasistencia a clases, mientras que ausentismo voluntario es la inasistencia a clase sin excusa válida. Ambos fenómenos se observan en estudiantes de medicina a nivel mundial y a menudo se asocian a una conducta inapropiada y un pobre desempeño académico. Sin embargo, se desconoce el comportamiento de éstos en la Facultad de Medicina de la Universidad de Panamá. Los objetivos de este estudio son: establecer la prevalencia del ausentismo voluntario, sus factores relacionados y la relación entre este y el índice académico.

Métodos y Materiales: Estudio descriptivo transversal de prevalencia en estudiantes de medicina de los ciclos básico y preclínico de la Universidad de Panamá. Se evaluó una muestra aleatoria sistemática, en conglomerados por semestre $(p=757, n=255)$. El análisis estadístico se realizó mediante $\mathrm{X}^{2}$ de Pearson.

Resultados: Se encontró que $75.98 \%$ de los estudiantes se ausentaban a clases voluntariamente y $59.06 \%$ se ausentaban en su mayoría previo a un examen, con incremento de éste a medida que se asciende de semestre. Destacaron como causales principales del ausentismo, el estudiar y dormir. No encontramos relación del ausentismo con el índice académico; no obstante el grupo de mayor índice registró menor ausentismo al realizar un punto de corte en el índice de 2.0/3.0.

Conclusión: El ausentismo voluntario tiene una alta prevalencia en nuestra facultad, incrementándose conforme se avanza de semestre. Estudiar y dormir fueron las causas principales. No se encontró diferencia en la prevalencia de ausentismo entre sexos. Tampoco se encontró alguna relación entre el ausentismo y el índice académico de manera general, sin embargo, los estudiantes de mayor índice registraron menor ausentismo si tomamos como punto de corte 2.0/3.0.

\section{ABSTRACT}

Introduction: Student absenteeism is class non-attendance, while voluntary absenteeism is student absenteeism without a valid excuse. Both phenomena are observed in medical students worldwide, but the behavior of these are unknown in the Faculty of Medicine of the University of Panama. The objectives are to establish the prevalence of voluntary absenteeism, its related factors and the relationship between voluntary absenteeism and the academic index.

Methods and Materials: Descriptive cross-sectional prevalence study in medical students during the basic and preclinical cycles in the University of Panama. The population is conformed by a systematic random sample in conglomerates by semester $(p=757, n=255)$. Statistical analysis was made using Pearson's $\mathrm{X}^{2}$.

Results: It was found that $75.98 \%$ of the students were voluntarily not attending to class and $59.06 \%$ were absent mostly prior to an exam; with an augmentation of this percentage as the semester increased. Sleep and study were emphasized among the reasons for absenteeism. We did not find an association between absenteeism and the academic index, but by establishing a cut-off point in the index of 2.0/3.0, it was found that the group with the highest index had less absenteeism.

Conclusion: Voluntary absenteeism in the Faculty of Medicine of the University of Panama has a high prevalence; accentuated as the semester increased. Study and sleep were the most common reasons. Higher academic index students had less voluntary absenteeism if we take a 2.0/3.0 cut-off point.

Key Words: Absenteeism, medical student, medical school.

Factores Asociados al Ausentismo Voluntario y su Relación con el Rendimiento Académico en Estudiantes de Medicina de la Universidad de Panamá by Autores is licensed under a Creative Commons Attribution-

NonCommercial-NoDerivs 3.0 Unported License.

Permissions beyond the scope of this license may be available at www.revistamedicocientifica.org. 


\section{INTRODUCCIÓN}

El ausentismo escolar o estudiantil se define como: "situación de inasistencia a clases por parte del alumno en la etapa obligatoria, de manera permanente y prolongada" ${ }^{1}$ debido a factores inevitables (enfermedades), factores personales (interés en la materia, motivación, vida social), factores relacionados con el curso (hora de la clase, dificultad del material, calidad y estilo de enseñanza, disponibilidad de apuntes del curso, tiempo del año). ${ }^{2}$ Por otra parte, en este estudio utilizamos como variable "ausentismo intencional", la cual hace referencia a la inasistencia a clases por parte del alumno de forma premeditada para realizar otras actividades no relacionadas a factores inevitables. Dicha definición se especificó en el instrumento de recolección de datos.

Algunos estudios revelan que las mujeres se ausentan más que los varones durante el periodo pre-examen, mientras que en periodos de clases el ausentismo tiene mayor prevalencia en estudiantes del sexo masculino. ${ }^{3,4,5}$ En una encuesta realizada por Field, S. ${ }^{6}$, el $84 \%$ de los 38 encuestados respondieron que han sido o pueden sentirse inclinados a faltar a una clase por motivos relacionados con la "salud": estar cansados o simplemente no sentirse bien. Todas las mujeres eligieron estas opciones mientras que solo el $67 \%$ de los hombres lo hizo. Más allá de las líneas de género, también influyó en las respuestas el año de la universidad: mientras que el 50\% de tercer y cuarto año eligió el "tiempo" como una razón para faltar a clase, sólo el $20 \%$ de los estudiantes de primer año y segundo año lo hizo. Esto sugiere que los profesores deberían analizar las políticas de asistencia en cuanto a género y año para considerar dar incentivos por la asistencia.

Sin embargo, se ha percibido con bastante claridad que muchos factores influyen en esto, incluyendo la institución educativa, la calidad de la enseñanza del instructor y el efecto residual que conlleva faltar a una clase sobre las clases subsecuentes. Las principales razones reportadas para clases faltantes es que se preparaban para un nuevo examen, falta de interés, el estilo de enseñanza del profesor, etc. Otros estudios realizados en estudiantes de medicina encontraron que el principal motivo de ausentismo era la preparación para exámenes cercanos. 7, ${ }^{8}$ Estudios también indican que la principal razón válida para ausentarse a clases es porque el estudiante se siente mal, pero no lo suficiente como para ir al doctor. Adicionalmente también se ha estudiado y demostrado una relación entre el ausentismo a clases, la edad del estudiante y el uso de drogas sociales. ${ }^{9,10}$

Por otra parte, el tema de la influencia del ausentismo en el desempeño académico de los estudiantes es un tema muy debatido. Algunas investigaciones han mostrado una asociación significativa entre el desempeño académico y el ausentismo estudiantil en los estudiantes de primer año. También revelan estos trabajos, que los estudiantes con mejor desempeño académico tenían menor ausentismo que los estudiantes con un peor desempeño académico, lo que indica una correlación negativa entre el rendimiento académico y el porcentaje de ausentismo a clases. ${ }^{11,12,13}$

En el estudio de Yusoff $(2014)^{12}$ realizado en la University Sains Malaysia en Malasia, se encontró una asociación significativa entre el desempeño académico y el ausentismo estudiantil en los estudiantes de primer año. Encontraron que los estudiantes que aprobaron los exámenes tenían una puntuación de ausentismo de 0.04 mientras que los estudiantes que reprobaron el examen tenían una puntuación de ausentismo de 9.0. En este estudio se comprobó que los estudiantes con mejor desempeño académico tenían menor ausentismo que los estudiantes con un peor desempeño académico.

Podemos considerar el ausentismo estudiantil como un tipo de conducta que afecta a la sociedad en general como consecuencia de distintos factores como motivos sociales, económicos, psicológicos, personales, entre otros. Se suele considerar al ausentismo como un mal social que lleva a la formación de personas irresponsables. La falta de asistencia también tiene que ver con otros factores estrictamente ligados al proceso educativo. Actualmente, la afluencia de información a través de dispositivos electrónicos, medios de comunicación y demás fuentes permite que los estudiantes universitarios tengan mayor acceso a distintos materiales de estudio $y$ facilitando el trabajo desde casa y haciendo que factores como las grandes distancias entre la casa y la facultad contribuyan de forma negativa a la asistencia a clase. ${ }^{14-16}$

Este estudio contiene resultados que podrían proveer información crítica y necesaria para el desarrollo de un plan académico adecuado, que motive a los estudiantes a dar su mejor desempeño académico con calificaciones satisfactorias sin tener que agotarlos ni promover su desinterés por los estudios. 
Así pues, el objetivo de este estudio es establecer la prevalencia del ausentismo intencional en los ciclos básico y preclínico de la Facultad de Medicina de la Universidad de Panamá, sus factores relacionados y si se evidencia alguna relación entre el ausentismo intencional y el índice académico.

\section{MATERIALES Y METODOS}

Este estudio fue desarrollado en la Universidad de Panamá, universidad estatal de la República de Panamá y una de las principales del país. Fundada en 1935, cuya ubicación es en el Cangrejo, Corregimiento de Bella Vista, Panamá, Panamá, Campus Ciudad Universitaria Octavio Méndez Pereira. ${ }^{18}$ Específicamente en la Facultad de Medicina.

El diseño de estudio es de tipo descriptivo transversal o de corte. Se aplicaron cuestionarios a estudiantes seleccionados de manera aleatoria por conglomerados en semestres, entre los seis primeros semestres de la carrera de Medicina de la Universidad de Panamá que constituye el ciclo básico y preclínico. Los cuestionarios fueron autoadministrados, mientras el aplicador del cuestionario supervisó el llenado de los mismos. Las variables estudiadas fueron edad, sexo, ausentismo estudiantil (medido como horas de clase faltadas durante el curso del actual semestre: 1-2, 3-5, 6-8, 9-11, más de 11) e índice académico (medido de dos maneras $\rightarrow 1$. en rangos: 1.0-1.25, 1.25-1.50, 1.50-1.75, 1.75-2.0, 2.0-2.5, 2.5-3.0; $\rightarrow 2$. Con un punto de corte: índice $\geq 2.0$, índice $<2.0$ ).

Las principales variables del estudio son el ausentismo estudiantil y el índice académico como medida de rendimiento académico. Estas variables principales se comparan con diversos factores sociodemográficos que según estudios previos relacionados pueden influir sobre el fenómeno de ausentismo estudiantil.

El universo del estudio está conformado por todos los estudiantes que cursan el ciclo básico o pre-clínico de la carrera de Medicina en la Facultad de Medicina de la Universidad de Panamá durante el segundo semestre del 2016. El ciclo básico incluye los estudiantes que cursan el primer o segundo semestre, el ciclo pre-clínico incluye los estudiantes que cursan el tercer, cuarto, quinto o sexto semestre de la carrera de Medicina en la Facultad de Medicina de la Universidad de Panamá. El universo estaba conformado por 750 estudiantes. El cálculo de la muestra se realizó utilizando el STAT CALC de EPI INFO $^{\text {TM }}$, en el cual resultó un total de 255 estudiantes agrupados por salón para tener un 95\% de nivel de confianza, una frecuencia esperada de $50 \%$ y un margen de error de $5 \%$. El muestreo fue aleatorio sistematizado proporcional al tamaño de cada grupo. La recolección de datos se realizó en sus aulas de clases garantizando la correcta estratificación de la muestra.

Criterios de Inclusión: Estudiantes que cursen entre el primer y el sexto semestre de la carrera de medicina en la Facultad de Medicina de la Universidad de Panamá. Los que consientan en participar.

Criterios de Exclusión: Estudiantes que formen parte del equipo encargado de realizar el estudio. Estudiantes que no estén tomando todas las materias del semestre correspondiente.

El instrumento utilizado para la recolección de los datos fue una encuesta de tipo cuestionario, compuesta primordialmente de preguntas cerradas. Ésta se validó interna y externamente por panel de expertos de epidemiólogos y docentes de la Universidad de Panamá. Además, se realizó una prueba piloto en un grupo de 15 estudiantes previo a su aplicación por los investigadores para asegurar la correcta recolección de los datos y calidad de los resultados. Dichos cuestionarios no fueron incluidos dentro de los resultados. Las respuestas del cuestionario fueron de carácter anónimo y con previo consentimiento informado. Para el análisis y presentación de los datos se utilizaron los siguientes programas: Microsoft Word 2013, Microsoft Excel 2013, Epi Info 7.3.5.; se realizó estadística descriptiva y prueba de $X^{2}$ para todos los análisis con un nivel de significancia de $95 \%$.

\section{RESULTADOS}

De la población estudiada 64\% (163) eran mujeres mientras que $36 \%$ (91) correspondía a hombres. La media de edad fue 19.65 \pm 1.36 años. En la muestra estudiada $20 \%$ (51) cursan el primer semestre, $22 \%(56)$ el segundo semestre, $19 \%$ (47) el tercer semestre, $18 \%$ (45) el cuarto semestre, $7 \%$ (19) el quinto semestre y $14 \%$ (36) el sexto semestre de la carrera de medicina.

De todos los estudiantes en el estudio, un 75,98\% (193) se ausenta a clases de manera voluntaria mientras que el $24,02 \%$ (61) no. También encontramos que el porcentaje de estudiantes que se ausentan en su mayoría previo a un examen fue de 59.06\% (150) mientras que $40.09 \%$ (104) de los estudiantes no se ausentan en su mayoría previo a un examen.

En cuanto a las horas faltadas a clase durante el curso del actual semestre, la mayoría de los estudiantes habían faltado 0 horas 
de clase (61), 60 estudiantes habían faltado entre 3-5 horas de clase, 37 habían faltado entre 1-2 horas de clase, 36 habían faltado entre 6-8 horas de clase, 32 habían faltado más de 11 horas de clase y 28 habían faltado entre 9-11 horas de clase.

En base al porcentaje de ausencia voluntaria por semestre se encontró el mayor porcentaje de ausencias voluntarias en el sexto semestre en donde $94.44 \%$ (34) se ausenta a clases de manera voluntaria mientras $5.56 \%$ (2) no, seguido por quinto semestre donde un $84.21 \%$ (16) se ausentaban voluntariamente mientras que el $15.79 \%$ (3) no, luego tercer semestre en donde $74.47 \%$ (35) se ausentaban voluntariamente y $25.53 \%$ (12) no, después primer semestre en el cual $72.55 \%$ (37) se ausentaba voluntariamente y $27.45 \%$ (14) no, luego cuarto semestre en donde $71.11 \%$ (32) se ausentaban de manera voluntaria mientras que $28.89 \%$ (13) no y finalmente segundo semestre en donde $69.64 \%$ (39) de los estudiantes se ausentaban voluntariamente mientras que el $30.36 \%$ (17) restante no (Figura 1).

Figura 1: Ausentismo voluntario por semestre en estudiantes de medicina de la Universidad de Panamá II semestre 2016.

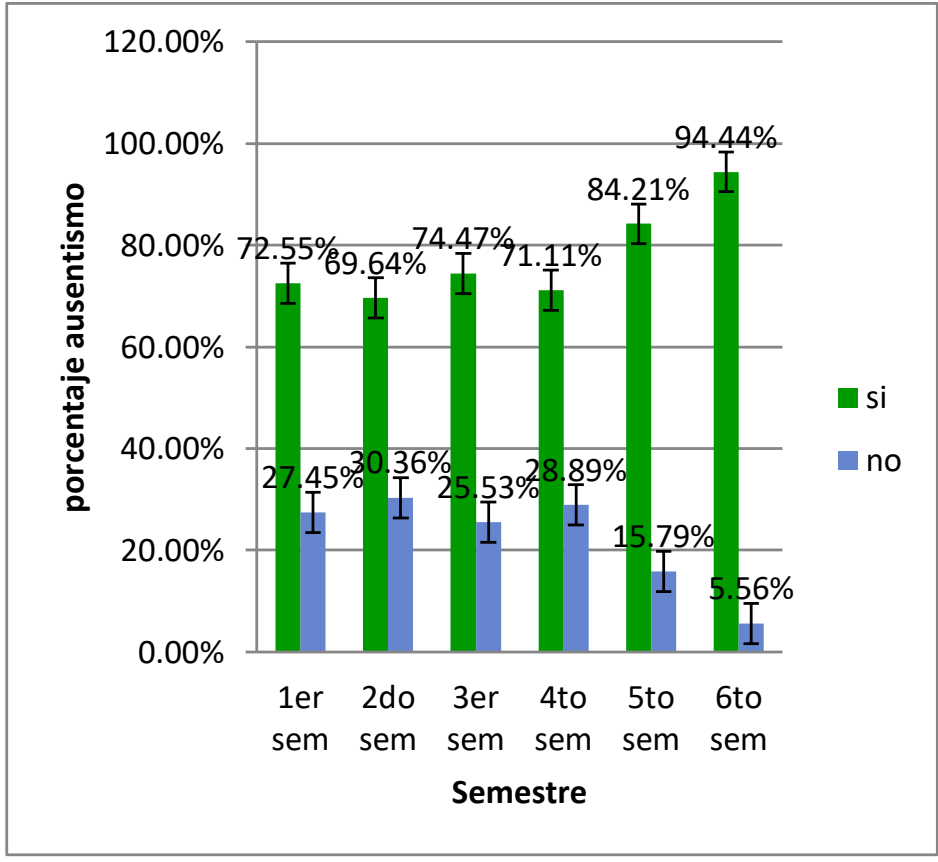

Fuente: Encuestas a los estudiantes de Medicina de la Universidad de Panamá.

Adicionalmente evaluamos la opinión de los estudiantes con respecto al ausentismo y encontramos que $69.69 \%$ (177) de los estudiantes opinan que ausentarse es un beneficio para estudiar y 30.31\% (77) opinan que no lo es. Se encontró que
$30.10 \%$ (79) opinan que ausentarse afecta el rendimiento académico, $34.25 \%$ (87) opinan que no y $34.65 \%$ (88) opinan que a veces.

En relación a los motivos del ausentismo, donde los estudiantes podían elegir múltiples opciones, encontramos que $49.63 \%$ (133) se ausentan por estudiar, $22.01 \%$ (59) por dormir, $10.07 \%$ (27) porque consideran que la materia no es interesante, $7.84 \%$ (21) porque no les gusta el profesor, $7.09 \%$ (19) porque tienen que hacer encargos, y $3.34 \%$ (9) listaron otras razones (Figura 2).

Figura 2: Motivos de ausentismo en estudiantes de medicina de la Universidad de Panamá II semester 2016

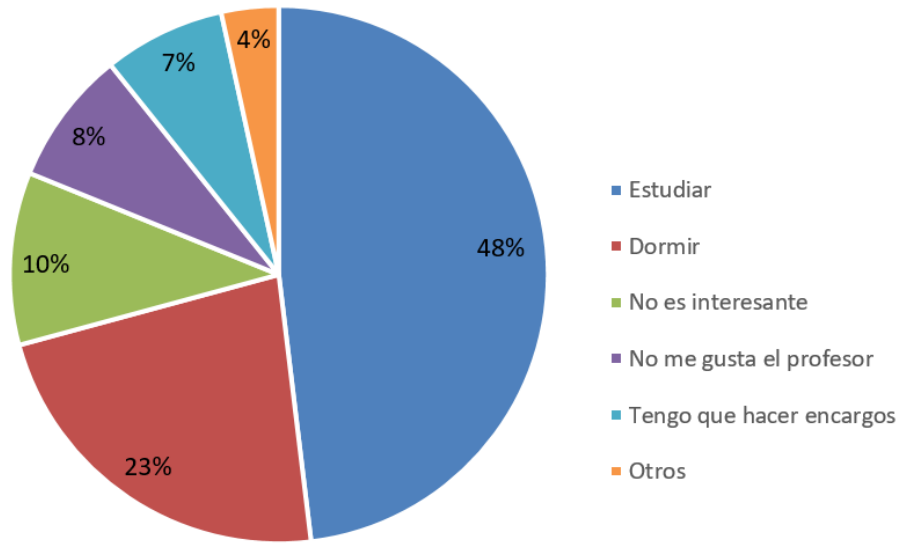

Fuente: Encuestas a los estudiantes de Medicina de la Universidad de Panamá.

En relación a los motivos de ausentismo previo a un examen se encontró que de los estudiantes de primer semestre $72.41 \%$ (21) se ausentan para estudiar, $17.24 \%$ (5) para dormir y $10.34 \%$ (3) porque no les parece interesante la clase. Encontramos que $47.27 \%$ (26) de los estudiantes de segundo semestre se ausentan para estudiar, $16.36 \%$ (9) para dormir, $16.36 \%$ (9) porque no les parece interesante la clase, $10.91 \%$ (6) porque no les gusta el profesor, $7.27 \%$ (4) porque tienen que hacer encargos y $1.82 \%$ (1) porque están enfermos.

Obtuvimos que de los estudiantes de tercer semestre $68.09 \%$ (32) se ausentan para estudiar, $14.89 \%$ (7) para dormir, $10.64 \%$ (5) porque no les parece interesante la clase y $6.38 \%$ (3) porque no les gusta el profesor. De los estudiantes de cuarto semestre, se encontró que $75.00 \%$ (24) se ausentan para estudiar, $21.88 \%$ (7) para dormir y $3.13 \%$ (1) porque llegan tarde. 
Se obtuvo que de los estudiantes de quinto semestre $92.31 \%$ (12) se ausentan para estudiar y $7.69 \%$ (1) porque no les parece interesante la clase. Los estudiantes de sexto semestre $68.42 \%$ (26) se ausentan para estudiar, $18.42 \%$ (7) para dormir, $7.89 \%$ (3) porque no les gusta el profesor, $2.63 \%$ (1) porque la clase es muy temprano y $2.63 \%$ (1) porque la materia no les parece interesante.

En base a lo presentado, se observa que la mayor causa de ausentismo tanto general como antes de un examen y para todos los semestres es estudiar. En segundo lugar en ambos general y previo a un examen se encuentra dormir, con la excepción de quinto semestre donde "no me parece interesante la clase" es la segunda causa de ausentismo previo a un parcial.

En cuanto a las materias a las cuales los estudiantes se ausentan, encontramos que en primer semestre $31.71 \%$ (13) de los estudiantes se ausentan en Historia de Panamá, 24.39\% (10) se ausentan en Teoría de la Ciencia, $12.20 \%$ (5) se ausentan en Español, $12.20 \%$ (5) en Química y $19.52 \%$ (8) en otras materias. Se obtuvo que en segundo semestre $22.03 \%$ (13) de los estudiantes se ausentan en Metodología de la Investigación, $16.95 \%$ (10) se ausentan en Biofísica, $13.56 \%$ (8) se ausentan en Química Orgánica, $13.56 \%$ (8) en Sociedad Medio Ambiente y Desarrollo y $33.89 \%(20)$ en otras materias.

En tercer semestre se encontró que $44.68 \%$ (21) de los estudiantes se ausentan en Bioquímica, $25.53 \%$ (12) se ausentan en Anatomía, 17.02\% (8) se ausentan en Embriología y $12.77 \%$ (6) en Realidad Social. En cuarto semestre $28.26 \%$ (13) de los estudiantes se ausentan en Microbiología, 26.09\% (12) se ausentan en Neuroanatomía, 23.91\% (11) se ausentan en Histología y $21.74 \%$ (10) en otras materias.

Se obtuvo que en quinto semestre $27.27 \%$ (6) de los estudiantes se ausentan en Fisiología, 22.73\% (5) Parasitología, 18.18\% (4) en Antropología y $31.82 \%$ (7) en otras materias. En sexto semestre $39.22 \%$ (20) de los estudiantes en Medicina Preventiva II, 29.41\% (15) en APS, $17.65 \%$ (9) en Patología, $11.76 \%(6)$ en otras materias y $1.96 \%$ (1) en todas las materias.

En la sección inferencial del estudio, buscamos asociación entre la variable principal (ausentismo voluntario) y sus distintas determinantes. Los resultados se resumen en la Tabla 1.
Tabla 1. Resumen de la estadística inferencial

\begin{tabular}{|c|c|c|}
\hline Variable estudiada & $\begin{array}{l}\text { Prueba } \\
\text { utilizada }\end{array}$ & $\begin{array}{l}\text { Resultado } \\
(2 \quad \text { colas, } \\
\text { corregido) }\end{array}$ \\
\hline $\begin{array}{l}\text { Prevalencia de ausentismo } \\
\text { voluntario en hombres vs } \\
\text { mujeres }\end{array}$ & $\begin{array}{l}X 2 \\
\alpha=95 \%\end{array}$ & $p=0.4708$ \\
\hline $\begin{array}{l}\text { Prevalencia de ausentismo } \\
\text { voluntario previo a un } \\
\text { examen en hombres vs } \\
\text { mujeres. }\end{array}$ & $\begin{array}{l}X 2 \\
\alpha=95 \%\end{array}$ & $p=0.2592$ \\
\hline $\begin{array}{l}\text { Ausentismo voluntario por } \\
\text { semestre }\end{array}$ & $\begin{array}{l}X 2 \\
\alpha=95 \%\end{array}$ & $p=0.0862$ \\
\hline $\begin{array}{l}\text { Ausentismo voluntario } \\
\text { antes de examen por } \\
\text { semestre }\end{array}$ & $\begin{array}{l}X 2 \\
\alpha=95 \%\end{array}$ & $p=0.0036 *$ \\
\hline $\begin{array}{l}\text { Ausentismo voluntario vs } \\
\text { índice académico (global) }\end{array}$ & $\begin{array}{l}X 2 \\
\alpha=95 \%\end{array}$ & $p=0.1292$ \\
\hline $\begin{array}{l}\text { Ausentismo voluntario } \\
\text { previo a examen vs índice } \\
\text { académico (global) }\end{array}$ & $\begin{array}{l}X 2 \\
\alpha=95 \%\end{array}$ & $p=0.1235$ \\
\hline $\begin{array}{l}\text { Ausentismo voluntario vs } \\
\text { índice académico (punto de } \\
\text { corte } \geq 2.0 \text { ) }\end{array}$ & $\begin{array}{l}X 2 \\
\alpha=95 \%\end{array}$ & $p=0.0393 *$ \\
\hline $\begin{array}{l}\text { Horas de ausentismo vs } \\
\text { índice académico (punto de } \\
\text { corte } \geq 2.0 \text { ) }\end{array}$ & $\begin{array}{l}X 2 \\
\alpha=95 \%\end{array}$ & $p=0.0062 *$ \\
\hline $\begin{array}{l}\text { Ausentismo previo a } \\
\text { examen vs índice } \\
\text { académico (punto de corte } \\
\geq 2.0 \text { ) }\end{array}$ & $\begin{array}{l}X 2 \\
\alpha=95 \%\end{array}$ & $P=0.0306$ * \\
\hline $\begin{array}{l}\text { Prevalencia Ausentismo } \\
\text { voluntario vs prevalencia } \\
\text { ausentismo voluntario } \\
\text { antes del examen }\end{array}$ & $\begin{array}{l}X 2 \\
\alpha=95 \%\end{array}$ & $\mathrm{P}<<<0.05^{*}$ \\
\hline
\end{tabular}

*Resultados estadísticamente significativos a 95\% de nivel de confianza

Fuente: Encuestas a los estudiantes de Medicina de la Universidad de Panamá.

Se buscaba probar si había diferencia entre prevalencia de ausentismo voluntario en hombres vs mujeres, realizamos la prueba de chi-cuadrado, con resultado $x^{2}=0.520$. Dado que la $p$ resultante $p=0.1943$ es mayor a $p=0.05$ con un nivel de confianza de $95 \%$, se demuestra que el porcentaje de ausentismo voluntario no difería en función del género.

En relación a la diferencia entre prevalencia de ausentismo voluntario previo a un examen en hombres vs mujeres, realizamos la prueba de chi-cuadrado, con $x^{2}=1.2732$ como resultado. Como $p=0.1057$ es mayor que $p=0.05$ con un nivel 
de confianza de 95\%, se encontró que el porcentaje de ausentismo voluntario previo a un examen no difería en función del género.

En cuanto a las horas de clase faltadas durante el curso del actual semestre y el sexo, al realizar un chi-cuadrado donde $x^{2}$ $=2.1921$ obtuvimos como resultado una $p=0.8220$. Siendo $p=$ 0.8220 mayor que $p=0.05$, con un nivel de confianza de $95 \%$, se concluye que no hay diferencia entre las horas de clase faltada y el sexo. De igual forma encontramos que al realizar un chicuadrado para ausentismo voluntario por semestre, siendo $x^{2}=$ 9.6362 y obteniendo como resultado $p=0.0862$; como $p=$ 0.0862 es mayor que $p=0.05$, con un nivel de confianza de $95 \%$, concluimos que no hay diferencia en el ausentismo voluntario por semestre.

También se realizó un chi-cuadrado para ausentismo previo a un examen por semestre y obtuvimos que siendo $x^{2}=17.5448$ y $p=0.0036$, como $p=0.0036$ es menor que $p=0.05$ con un nivel de confianza de $95 \%$, se concluye que si hay diferencia entre las ausencias previas a un examen por semestre. Siendo quinto y sexto los semestres con mayor porcentaje de ausentismo previo a un examen (Figura 3).

Figura 3: Ausentismo previo a un examen por semestre en estudiantes de medicina de la Universidad de Panamá II semestre 2016

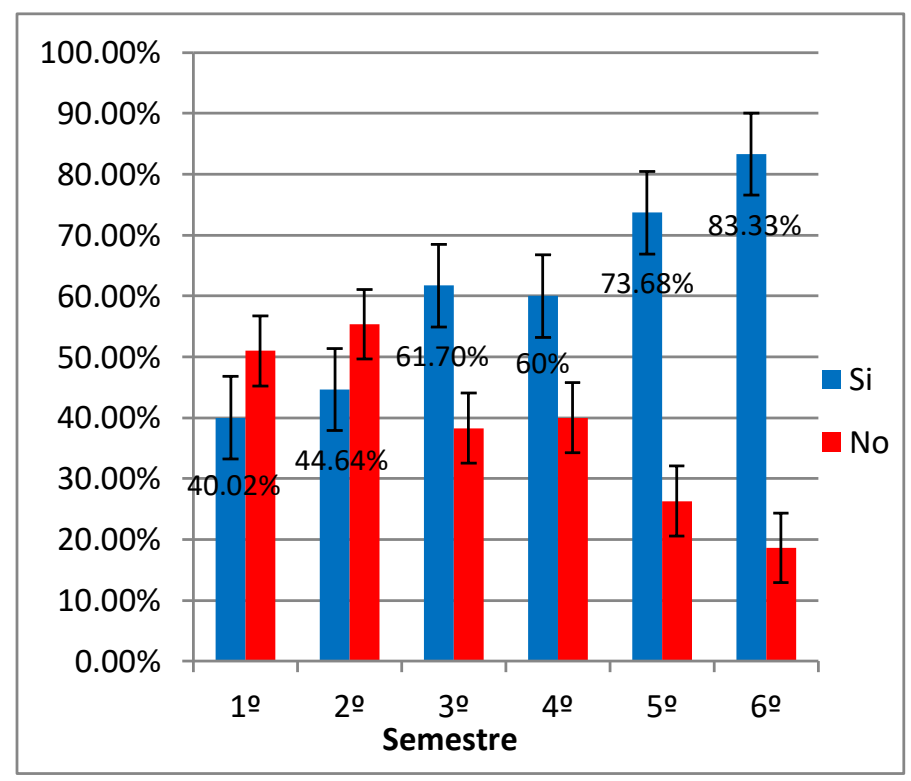

Fuente: Encuestas a los estudiantes de Medicina de la Universidad de Panamá.
Se realizó un chi-cuadrado para conocer la influencia del ausentismo voluntario sobre el índice académico, obteniéndose $x^{2}=7.1302$ y $p=0.1292$. Como $p=0.1292$ es mayor que $p=0.05$ con un nivel de confianza de $95 \%$, podemos concluir que el ausentismo voluntario no difiere con el índice académico, es decir que no influye sobre este. $Y$ al realizar un chi-cuadrado para determinar la influencia del ausentismo previo a examen sobre el índice académico, resultó $x^{2}=7.2454$ y $p=0.1235$. Siendo $p=0.1235$ mayor que $p=0.05$ con un nivel de confianza de $95 \%$, se concluye que no hay diferencia entre ausencia previa a un examen y el índice académico, es decir que tampoco influye sobre este.

Ante los resultados obtenidos, decidimos transformar los rangos del índice académico en una variable dicotómica con punto de corte, definida como índice académico $\geq 2.0$ y volver a realizar una prueba estadística de $X^{2}$ para determinar si había diferencia significativa entre estos grupos con relación al ausentismo voluntario.

El $X^{2}=4.2464$, resultado una $\mathbf{p}=\mathbf{0 . 0 3 9}$. Como $p=0.039$ es menor que $p=0.05$ con un nivel de confianza de $95 \%$, se concluye que si hay diferencia significativa entre ambos grupos de índice $\geq 2.0$ e índice $<2.0$ con respecto al ausentismo voluntario. Según lo que podemos observar en la tabla y los cálculos de OR, los estudiantes que practican el ausentismo voluntario tienen 2.1652 veces más probabilidades de tener índice académico $<2.0$.

Luego se investigó si existe diferencia entre la frecuencia del ausentismo voluntario y el resultado previamente obtenido con el índice $\geq 2.0$ y si variaba al compararlo en periodos previos a examen.

Para esto, se realizó un chi-cuadrado con $x^{2}=16.2179$ resultando una $p=0.0062$. Como $p=0.0062$ es mayor que $p=$ 0.05 con un nivel de confianza de $95 \%$, se concluye que hay diferencia entre la cantidad de horas de clases faltadas y tener un índice académico $\geq 2.0$.

Dado que el $\mathrm{X}^{2}$ solo permite saber si hay diferencias significativas entre los grupos estudiados, no es posible en este punto determinar desde que cantidad de horas faltadas se evidencia esa diferencia significativa. En las horas de clase faltada solo se demuestra diferencia estadísticamente significativa en la cantidad de horas faltadas entre los grupos con índice $\geq 2.0$ y aquellos que no. No evidencia el 
comportamiento lineal o inverso, pero según lo encontrado en el estudio se ve una tendencia de aquellos con índice $\geq 2.0$ de faltar a menos horas de clase de forma voluntaria (Figura 4).

Figura 4. Tabla de análisis estadístico $X^{2}$ para horas de clase faltada por estudiantes de Medicina de la Universidad de Panamá II semestre 2016.

\begin{tabular}{|c|c|c|c|}
\hline \multirow[b]{2}{*}{ Horas de clase faltada } & \multicolumn{3}{|c|}{ Indice $\geq 2.00$} \\
\hline & $\mathrm{Si}$ & No & Total \\
\hline$o$ & $\begin{array}{l}20 \\
(42.55 \%) \\
(33.90 \%)\end{array}$ & $\begin{array}{l}27 \\
(57.45 \%) \\
(19.15 \%)\end{array}$ & $\begin{array}{l}47 \\
(100.00 \%) \\
(23.50 \%)\end{array}$ \\
\hline $1-2$ & $\begin{array}{l}11 \\
(45.83 \%) \\
(18.64 \%)\end{array}$ & $\begin{array}{l}13 \\
(54.17 \%) \\
(9.22 \%)\end{array}$ & $\begin{array}{l}24 \\
(100.00 \%) \\
(12.00 \%)\end{array}$ \\
\hline $3-5$ & $\begin{array}{l}9 \\
(20.45 \%) \\
(15.25 \%)\end{array}$ & $\begin{array}{l}35 \\
(79.55 \%) \\
(24.82 \%)\end{array}$ & $\begin{array}{l}44 \\
(100.00 \%) \\
(22.00 \%)\end{array}$ \\
\hline $6-8$ & $\begin{array}{l}2 \\
(7.41 \%) \\
(3.39 \%)\end{array}$ & $\begin{array}{l}25 \\
(92.59 \%) \\
(17.73 \%)\end{array}$ & $\begin{array}{l}27 \\
(100.00 \%) \\
(13.50 \%)\end{array}$ \\
\hline $9-11$ & $\begin{array}{l}6 \\
(22.22 \%) \\
(10.17 \%)\end{array}$ & $\begin{array}{l}21 \\
(77.78 \%) \\
(14.89 \%)\end{array}$ & $\begin{array}{l}27 \\
(100.00 \%) \\
(13.50 \%)\end{array}$ \\
\hline Más de 11 & $\begin{array}{l}11 \\
(35.48 \%) \\
(18.64 \%)\end{array}$ & $\begin{array}{l}20 \\
(64.52 \%) \\
(14.18 \%)\end{array}$ & $\begin{array}{l}31 \\
(100.00 \%) \\
(15.50 \%)\end{array}$ \\
\hline Total & $\begin{array}{l}59 \\
(29.50 \%) \\
(100.00 \%)\end{array}$ & $\begin{array}{l}141 \\
(70.50 \%) \\
(100.00 \%)\end{array}$ & $\begin{array}{l}200 \\
(100.00 \%) \\
(100.00 \%)\end{array}$ \\
\hline
\end{tabular}

Fuente: Encuestas a los estudiantes de Medicina de la Universidad de Panamá.

En cuanto a las ausencias previas a un examen y el índice académico, el $X^{2}=4.6744$, resultó una $p=0.0306$. Como $p=$ 0.0306 es menor que $p=0.05$ con un nivel de confianza de $95 \%$, se concluye que si hay diferencia significativa entre ambos grupos de índice $\geq 2.0$ e índice $<2.0$ con respecto al ausentismo voluntario previo a un examen. Según lo que podemos observar en la tabla y los cálculos de OR, los estudiantes que practican el ausentismo voluntario previo a un examen tienen 2.0690 veces más probabilidades de tener índice académico $<2.0$.

Por último, al comparar el ausentismo previo a un examen con el ausentismo voluntario, obtuvimos resultados que indican que $p=0.0001$, siendo esta menor que $p=0.05$, se observa diferencia significativa entre ausentismo voluntario $y$ el ausentismo previo a un examen. Al regresar a la tabla $2 \times 2$ del análisis estadístico, se observa que la mayor frecuencia de ausencias se da en periodos previos a un examen.

\section{DISCUSIÓN}

La prevalencia general estimada del ausentismo voluntario en la Facultad de Medicina de la Universidad de Panamá en estudiantes del ciclo básico y preclínico resultó ser del $75.98 \%$ según este estudio, sin embargo esta medida no refleja la frecuencia de dicho ausentismo, ya que los resultados obtenidos muestran que el $38.19 \%$ de los que se han ausentado voluntariamente lo han hecho solo 1-5 horas de clase.

Esos resultados parecen ser alentadores, sin embargo están enmascarados al tomar toda la población, ya que se observan diferencias entre los semestres. Particularmente a lo largo del análisis de las distintas variables se observa una tendencia al incremento de las horas de clase faltada a medida que aumenta el semestre. Posteriormente se aplicó una prueba de hipótesis para determinar si había diferencia estadística entre semestres y a la única conclusión estadísticamente significativa que podemos llegar es que hay diferencia entre la prevalencia del ausentismo previo a examen entre semestres, con las prevalencias más altas en quinto y sexto semestre.

En cuanto a los motivos principales de ausentismo comprobamos rotundamente que en nuestra facultad el principal motivo de ausentismo voluntario es estudiar el cual asciende a un $49.63 \%$ de las respuestas. En la tabla 2 se denotan los motivos de ausentismo encontrados por los distintos estudios de referencia.

Tabla 2. Comparación de los motivos de ausentismo en estudios encontrados en la literatura.

\begin{tabular}{|c|c|c|c|c|}
\hline $\begin{array}{l}\text { Rodríguez }(2003)^{9} \\
\text { - universitarios }\end{array}$ & $\begin{array}{l}\text { Organización de la } \\
\text { clase/ metodología }\end{array}$ & Valor de la clase & $\begin{array}{l}\text { Actitud del } \\
\text { profesorado }\end{array}$ & $\begin{array}{l}\text { Proximidad de } \\
\text { exámenes o } \\
\text { falta de tiempo } \\
\text { para estudiar en } \\
\text { relación al } \\
\text { volumen de la } \\
\text { asignatura }\end{array}$ \\
\hline $\begin{array}{l}\text { Lang }(2008)^{2} \\
\text { - universitarios }\end{array}$ & Factores inevitables & $\begin{array}{l}\text { Factores } \\
\text { personales }\end{array}$ & $\begin{array}{l}\text { Factores } \\
\text { relacionados con } \\
\text { el curso }\end{array}$ & \\
\hline $\begin{array}{l}\text { Triado-Ivern, X., } \\
\text { Olmos, J., et al (2009) } \\
\text { 18.-universitarios }\end{array}$ & $\begin{array}{l}\text { Obtener el material } \\
\text { de las clases } \\
\text { semipresenciales }\end{array}$ & $\begin{array}{l}\text { Falta de } \\
\text { convivencia, de } \\
\text { mentores y } \\
\text { referentes, y de } \\
\text { ejemplos vivos. }\end{array}$ & $\begin{array}{l}\text { No } \\
\text { obligatoriedad } \\
\text { de asistencia a } \\
\text { clase. }\end{array}$ & \\
\hline $\begin{array}{l}\text { Ateneh, A., Asres, B., } \\
\text { et al (2014) } \\
\text {-medicina }\end{array}$ & $\begin{array}{l}\text { Preparaban para un } \\
\text { nuevo examen }\end{array}$ & Falta de interés & $\begin{array}{l}\text { Estilo de } \\
\text { enseñanza del } \\
\text { profesor }\end{array}$ & \\
\hline $\begin{array}{l}\text { Bati, A. (2003) }{ }^{10}- \\
\text { ciencias de la salud }\end{array}$ & $\begin{array}{l}\text { Insomnio, mala } \\
\text { salud }\end{array}$ & $\begin{array}{l}\text { Ineficacia de las } \\
\text { clases en salas } \\
\text { de hacinamiento }\end{array}$ & $\begin{array}{l}{ }^{*} \text { Estudiantes de } \\
\text { medicina mayor } \\
\text { índice de } \\
\text { inasistencia }\end{array}$ & \\
\hline $\begin{array}{l}\text { Kaushik T., Rituparna } \\
\text { D., et al }(2015)^{8}- \\
\text { medicina }\end{array}$ & $\begin{array}{l}\text { Estudiar para } \\
\text { exámenes }\end{array}$ & $\begin{array}{l}\text { Factores } \\
\text { relacionados a } \\
\text { los profesores }\end{array}$ & $\begin{array}{l}\text { Prefieren } \\
\text { estudiar en casa }\end{array}$ & \\
\hline
\end{tabular}

Fuente: Búsqueda bibliográfica.

Resultados similares a lo presentado en este estudio fueron obtenidos en el estudio realizado por Rodríguez, R, Hernández, J, et al, donde un $78.8 \%$ de los encuestados afirmaban no asistir a clase porque preferían dedicar el tiempo a estudiar. Esto es en parte debido a que muchas de las materias que se dan 
durante estos ciclos tienen un material muy extenso que cubrir durante un semestre, por lo que muchos estudiantes optan por ausentarse de sus clases teóricas para poder cubrir todo el material a su propio ritmo.

Es interesante denotar que el motivo principal de ausentismo (estudiar) no cambia a lo largo de los semestres. En todos y cada uno de los semestres evaluados el primer motivo es estudiar, seguido en la mayoría de los casos de dormir. El estudio mencionado anteriormente por Rodríguez, R, Hernández, J, et $\mathrm{al}^{9}$, también concluye que las principales razones por la cual los estudiantes se ausentan son: "metodología", la "proximidad de exámenes", la "falta de motivación por parte del profesor", y la "falta de tiempo para estudiar en relación al volumen de la asignatura". Esto es corroborado también en este estudio ya que el $69.69 \%$ de los estudiantes respondieron que consideran que es beneficioso para estudiar ausentarse a clases. Dicho hallazgo también coincide con la revisión realizada por $\mathrm{T}$ Sharmin, E Azim et al $(2016)^{15}$ y los estudios de Ahmed Alghamdi, Ahmed Yamani et al (2016). ${ }^{14}$

Como hemos examinado, el ausentismo intencional es un fenómeno un tanto complejo que es modificado por muchos factores, entre ellos con posibilidad el sexo. En la población estudiada hay un predominio claro del sexo femenino (64\%). En el estudio se buscaba demostrar como en estudios previos, alguna diferencia entre el ausentismo en hombres vs mujeres. Sin embargo, midiendo el ausentismo desde distintos puntos de vista (ausentismo en periodos regulares, ausentismo previo examen $y$ horas de clase faltada) en ninguno hallamos diferencia significativa entre hombres y mujeres. Según los resultados, en la Facultad de Medicina de la Universidad de Panamá, hombres y mujeres se comportan similares en cuanto a los patrones de ausentismo, a diferencia del estudio realizado en el 2007 por Abdulaziz A., Binsaeed M. ${ }^{4}$, et al, dirigido a los estudiantes de tercer año de la carrera de medicina, en el King Khaled University Hospital, Riyadhel en Arabia Saudita, se mostró que una mayor proporción de estudiantes de sexo masculino estaban ausentes en comparación a estudiantes de sexo femenino en todas las épocas del año académico. Sin embargo, el $68,7 \%$ de estudiantes del sexo femenino estaban ausentes durante el período pre-examen, en comparación con el $49,2 \%$ de los varones.

Uno de los comentarios más escuchados en los pasillos de la facultad y utilizados por muchos profesores es que al acercarse la fecha de parciales, los estudiantes tienden a ausentarse más.
Esto se comprueba con los resultados de este estudio. La prevalencia del ausentismo es estadísticamente mayor en periodos previos a un examen que en periodos regulares tomando toda la población de estudio. Resultados similares se encontraron en el estudio de Ahmed Alghamdi, Ahmed Yamani et al (2016)..$^{14}$

En cuanto a las materias con mayor ausentismo, se reporta lo siguiente: Teoría de la Ciencia $\left(1 .^{\circ}\right)$, Metodología de la Investigación $\left(2 .^{\circ}\right)$, Bioquímica $\left(3 .^{\circ}\right)$, Microbiología $\left(4 .^{\circ}\right)$ Fisiología $\left(5 .^{\circ}\right)$ y Medicina Preventiva y Social II $\left(6 .^{\circ}\right)$. Esta información no refleja el ausentismo verdaderamente ya que no se tomaron registros de asistencias pero funciona como medidor que puede ayudar a que los profesores busquen mecanismos para reducir el ausentismo.

Finalmente, era de interés conocer si en población de los ciclos básico y preclínico de la Facultad de Medicina de la Universidad de Panamá hay relación entre el ausentismo y el índice académico. Según los resultados midiendo el ausentismo desde distintos puntos de vista, en ninguno se halló diferencia significativa entre el grado de ausentismo y el índice académico. Sin embargo, al agrupar los índices académicos $\geq 2.0$ se encontró una diferencia estadísticamente significativa entre los grupos que se ausentan y los que no. También se observó que el ausentismo intencional aumenta 2.1652 veces las probabilidades de tener índice académico $<2.0$ y el ausentismo intencional previo a un examen aumenta 2.0690 veces más las probabilidades de tener índice académico <2.0. Resultados similares que concuerdan con la teoría de a mayor ausentismo menor rendimiento académico se pueden encontrar en los estudios de Jimenez, Rodríguez et al (2013) $)^{11}$ y Yussoff $(2014)^{12}$ y es explorado en la revisión de T Sharmin, E Azim et al $(2016)^{15}$, B.T.Rao, K. Valleswary (2016). ${ }^{16}$

\section{CONCLUSIÓN}

La prevalencia general estimada del ausentismo voluntario en la Facultad de Medicina de la Universidad de Panamá en estudiantes del ciclo básico y preclínico resultó ser del 75.98\% según este estudio. El ausentismo voluntario es una medida que utilizan los estudiantes en la Facultad de Medicina de la Universidad de Panamá para tratar de cubrir el material extenso que se coloca frente a sus ojos en cada semestre del ciclo básico y preclínico. En este estudio se comprobó que efectivamente hay una diferencia entre el ausentismo por semestre, con una 
tendencia a aumentar a medida que aumenta el semestre, lo cual es un reflejo de la creciente complejidad de la carrera y menos tiempo para estudiar, ya que el principal motivo de ausentismo resultó ser, como era lo esperado, para estudiar.

Según los resultados obtenidos, no parece haber diferencia entre hombres y mujeres en cuanto al ausentismo ni tampoco una relación entre el ausentismo y un mejor o peor índice académico de manera global, pero si una tendencia a tener índices académicos menor de 2.0 en aquellos que se ausentan más. Por ende, se considera la información presentada posee amplias aplicaciones en el planeamiento de los horarios y las cátedras para tratar de mejorar la calidad de vida del estudiante de medicina en los ciclos básico y preclínico.

\section{LIMITACIONES}

Este estudio cuenta con algunas limitaciones ya que es un cuestionario autoadministrado por el estudiante y por lo tanto se mantiene patente el posible sesgo de información. Adicionalmente, el instrumento de recolección de datos no fue validado de forma cuantitativa con una prueba estandarizada, sino solamente mediante validación por expertos y una prueba piloto. Se concluye que la limitación más importante, es que la variable del ausentismo representa en sí una dificultad en su definición y medición, con gran variabilidad en su medición en los distintos estudios.

Se recomienda la realización de otros estudios que aborden este tema y midan otras variables sociodemográficas no incluidas en este trabajo.

\section{AGRADECIMIENTOS}

Queremos agradecer a la Ingeniera Fermina Chamorro por guiarnos durante la elaboración del protocolo, el instrumento de recolección de datos y por todos los consejos de metodología. A nuestros compañeros Amarilis Abrego, Ana Barba, Sefali Ahir, Daphne Aizprua, Boris Bishop $\_$Alejandra Barahona y Keysi Bultrón por la ayuda con la recolección de datos y tabulación. A todos los estudiantes que nos dieron de su tiempo para completar las encuestas y hacer esta investigación posible.

Un agradecimiento especial a la Mgtr. Alleyne Thompson por su ayuda en la elaboración de la base de datos y su conversión a datos fácilmente leíbles por el programa estadístico.

\section{CONFLICTO DE INTERESES}

Algunos de los autores de este trabajo de investigación son editores en la Revista Médico Científica. No hay conflictos de intereses adicionales.

\section{REFERENCIAS BIBLIOGRÁFICAS}

1. Uruñuela P. Absentismo escolar [Internet]. Weib.caib.es. 2005 (consultado 10 Julio 2017). Disponible en: http://weib.caib.es/Documentacio/jornades/jornades_menors /p_urunuela.pdf

2. Lang, M., Joyce, A., Conaty, F. \& Kelly, B. An Analysis of Factors Influencing the Attendance of First Year University Students. In Pieterick, J. et al. (eds), Proceedings of European First Year Experience Conference, Wolverhampton, UK,2008, May 7-9, pp. 141-147.

3. Marburger D. Absentismo y desempeño en los exámenes a nivel de pregrado. The Journal of Economic Education [Internet]. 2001;32(2):99. Disponible en: https://econpapers.repec.org/article/tafjeduce/v_3a32_3ay_3 a2001_3ai_3a2_3ap_3a99-109.htm

4. Abdulaziz A. BinSaeed, Metib S. al-Otaibi, Hassan G. alZiyadi, AbdulMohsin A.Babsail \& Shaffi Ahamed Shaik, Asociación entre la ausencia de los estudiantes en la escuela de medicina y sus índices académicos. IAMSE - International Association of Medical Science Educators. Volumen 19: No.4. Disponible en: http://www.iamse.org/mse-article/associationbetween-student-absenteeism-at-a-medical-college-and-theiracademic-grades/

5. Anteneh Assefa Desalegn, Asres Berhan y Yifru Berhan, Ausentismo en estudiantes de pregrado en la escuela de medicina y otras ciencias de la salud de la Universidad de Hawassa, Etiopia. BMC Med Educ, publicado el 14 de abril de 2014. Acceso en línea: http://www.ncbi.nlm.nih.gov/pmc/articles/PMC3991889/

6. Field, S. Understanding attendance and nonattendance motivation amongst first year undergraduate students. Edge Hill University, 2012. Disponible en: https://www.edgehill.ac.uk/solstice/files/2012/06/Day-2Session-4-Will-this-Come-Up-in-the-Exam.pdf 
7. Álvarez, P.; López, D. Absenteeism in university education: an obstacle for the participation and self-guided work of students. 2011. Bordón 63 (3), 43-56.

8. Kaushik Tripura, Rituparna Das, Nirmalya Saha. (2015). Attitude of medical students towards the reasons of absenteeism in a medical college of Tripura. Noviembre 2015, de Journal of Dental and Medical Sciences. Disponible en: http://www.iosrjournals.org/iosr-jdms/papers/Vol14-

issue11/Version-5/U014115110112.pdf

9. Rodríguez, R., Hernández, J., Alonso, A., \& Diez-Itza, E. El absentismo en la Universidad: resultados de una encuesta sobre motivos que señalan los estudiantes para no asistir a clase. Aula Abierta,117-145. 2003.

10. Bati, A.H. Why do students miss lectures? A study of lectures attendance among students of health science. Nurse Educ Today. 2003;33(6): 596-601.Disponible en: http://www.peakjournals.org/journals/jers/archive/2015/Jan/ pdf/JERS-14-025\%20Dashputra\%20et\%20al\%20.pdf

11. Jiménez, J., \& Rodríguez, Á. El absentismo en las aulas universitarias. El caso de la Escuela Universitaria de Estudios Empresariales de la Universidad de Sevilla. Revista de Docencia Universitaria, 2013; 469-473.

12. Yusoff, S.B.M. Association of academic performance and absenteeism among medical students. Education in medicine Journal, 2014; 6(1), 40-44.

13. Dhaliwal, U. Absenteeism and under-achievement in final year medical students. Natl Med J India. 2003;16(1): 7-34

14. Ahmed Alghamdi, Ahmed Yamani, Ayman Khalil, Baraa Albarkati, Osama Alrehili, Mohamed Salih. Prevalence, causes and impacts of Absenteeism among medical students at UQU. Scientific and academic publishing [Internet]. 2016 [19 de enero de 2018]; 6(1): 9-12. Disponible en: http://article.sapub.org/10.5923.j.edu.20160601.02.html

15. TSharmin, E Azim, S Choudhury, S Kamrun. Reasons of Absenteeism among Undergraduate Medical Students: A Review. Awer Khan Modern Medical College Journal [Internet]. 2017[19 de enero de 2018];8(1): 60-66. Disponible en: https://www.banglajol.info/index.php/AKMMCJ/article/view/ 31660
16. B.T.Rao, K. Valleswary, M.Siva Durga Prasad Nayak, N. Lakshmana Rao. Reasons for Absenteeism among the Undergraduate Medical Students Attending for Theory Classes in Rajiv Gandhi Institute of Medical Sciences (RIMS) Ongole, Prakasam District of Andhra Pradesh: A Self Review. IOSR Journal of Research \& Method in Education [Internet]. 2016[20 de enero de 2018];6(4):11-19. Disponible en: https://www.researchgate.net/publication/305769326_Reaso ns_for_Absenteeism_among_the_Undergraduate_Medical_St udents_Attending_for_Theory_Classes_in_Rajiv_Gandhi_Instit ute_of_Medical_Sciences_RIMS_Ongole_Prakasam_District_of _Andhra_Pradesh_A_Self_Revie

17. Universidad de Panamá. Dirección de informática de la Universidad de Panamá. [Consultado 17 diciembre 2016] Disponible en: http://www.up.ac.pa/PortalUp/Historia.aspx.

18. Olmos, J, Et, A.L. Aproximación empírica al análisis del absentismo de los estudiantes universitarios. Revista de Formación e Innovación Educativa Universitaria. 2009;2(4): 182-192. 